Chapter 4

\title{
Cardiovascular Risk Assessment in Developing World
}

\author{
Parinya Chamnan and Wichai Aekplakorn \\ Additional information is available at the end of the chapter
}

http://dx.doi.org/10.5772/intechopen.69041

\begin{abstract}
Many international and national authorities recommend that cardiovascular risk assessment using multivariate risk scores be used to identify individuals at high risk of cardiovascular disease (CVD). This approach is likely to assure that resources in developing countries are allocated to those who need it most. However, not many developing countries have implemented this approach and different countries have varying progresses in adopting the concept. While many developing countries solely described estimated cardiovascular risk by applying existing CVD risk scores to their population's cross-sectional data, a number of countries have validated and recalibrated existing risk scores and only a few have developed new risk scores specific to their populations. To enhance the adoption of such a policy in developing countries, new CVD risk prediction charts for low- and medium-resource settings were developed and endorsed by the WHO and International Society of Hypertension. However, a number of issues need to be addressed, including development of population-specific risk scores, recalibration of available risk scores and uncertainty over cost-effectiveness of CVD risk assessment in developing countries. Although this high risk approach might represent an effective and practical strategy for developing countries, a complementary population-based approach is also needed to maximize benefits for CVD prevention.
\end{abstract}

Keywords: cardiovascular disease, risk assessment, prediction, primary prevention, developing countries

\section{Introduction}

Quantitative assessment of cardiovascular risk has become part of strategies for prevention of cardiovascular disease (CVD) in many countries [1,2]. Many international and national authorities have recommended that cardiovascular risk assessment using multivariate risk scores be used to identify individuals at high risk of cardiovascular disease, to whom preventive interventions can be targeted [3-6]. This high-risk approach has been advocated and 
routinely used in many developed countries [7, 8]. However, it has not been frequently used in developing countries. This chapter summarizes evidence on cardiovascular risk assessment in developing countries by (i) describing the overall rationale for using cardiovascular risk scores; (ii) systematically reviewing the literature on cardiovascular risk scores that have been developed, validated and/or practically used in developing countries; (iii) exploring issues surrounding the utility of the cardiovascular risk scores in these countries; and (iv) limitations and implications for alternative approaches.

\section{Concept and rationale for cardiovascular risk assessment}

Observational studies have shown a positive continuous association between traditional cardiovascular risk factors and CVD risk [9-11]. For example, results from the Prospective Studies Collaboration, a meta-analysis of almost 900,000 healthy men and women in 61 prospective observational studies, show a linear, continuous relationship between serum total cholesterol and the risk of death from ischemic heart disease. Similar associations were also held true for each of other traditional risk factors such as blood sugar [10] and blood pressure [11]. This underlines the importance of efforts to address each individual risk factor for prevention of cardiovascular disease.

However, there is evidence that clustering of risk factors confers higher levels of CVD risk than each individual risk factor. Evidence from observational prospective studies suggests that a small effect of an individual risk factor on CVD risk could be magnified in the presence of other risk factors $[10,12,13]$. In other words, an individual with mildly abnormal levels of several risk factors often has a greater absolute CVD risk than someone with a raised level of one risk factor. For example, data from the EPIC-Norfolk cohort revealed that CVD risk could vary up to 30 -fold in people with the same levels of traditional CVD risk factors, such as cholesterol and blood pressure, but different abnormalities in other risk factors [10] (Figure 1).

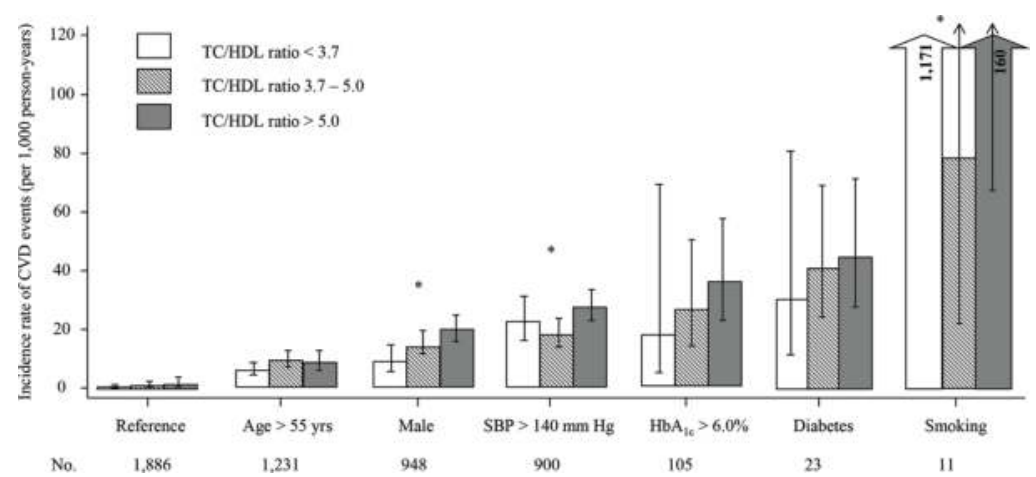

Figure 1. Absolute rates of cardiovascular events over 10 years in individuals with different levels of CVD risk factors in EPIC-Norfolk [10] ( $n=10,144)$, Reference group: non-smoking women aged $\leq 55$ years with a SBP of $\leq 140 \mathrm{~mm} \mathrm{Hg}$ and an $\mathrm{HbA}_{1 \mathrm{c}} \leq 6.0 \%,{ }^{*} p<0.001$, Reproduced with approval from Diabetologia. 
The combined effect of risk factors on absolute risk of CVD underlines the importance of using multivariate risk prediction tools.

Randomized controlled trials have confirmed that treatment with lipid-lowering medication reduces the risk of ischemic heart disease and stroke, regardless of pre-treatment blood cholesterol levels or other characteristics of the study participants. Overall, there was a 19\% reduction in coronary mortality for each $1 \mathrm{mmol} / \mathrm{L}$ reduction of low density lipoprotein (LDL) cholesterol [14]. Similarly, trial evidence suggests that blood pressure-lowering drugs reduce the risk of developing CVD events with comparable relative risk reductions at different pre-treatment levels of blood pressure [15]. This suggests that substantial benefits could be achieved from modifying these risk factors at any starting level in individuals whose absolute CVD risk is high for whatever aetiological reason. The concept of treating individuals with a disease diagnosed by a threshold of one single risk factor, e.g. diabetes, hypertension or hypercholesterolemia, has thus been challenged [12, 16].

Key question is how we can identify those at high CVD risk, which preventive interventions could be targeted. Compelling evidence points out that absolute risk assessment, based on the combined effect of multiple risk factors, is likely to be an approach of choice. Compared with multifactorial CVD risk assessment, major CVD risk factors such as blood pressure or blood lipid levels are, individually, poorer predictors of future CVD risk and of the benefits of treatment in individuals with and without existing CVD [1, 10, 16]. In addition, primary prevention informed by multifactorial CVD risk assessment is likely to prevent a similar number of cardiovascular events at a possibly lower cost than a singlefactor approach [17].

There are a number of reasons why it may be important to quantify the risk of developing cardiovascular disease (Table 1). The clear rationale for the development of a risk score is critical to how its validity is assessed and how it is used.

First, risk scores may be used to rank individuals and groups according to their absolute cardiovascular risk so that preventive interventions for those at greatest risk could be targeted $[1,7]$. Concerning this purpose, it is the ranking that is important and not necessarily the absolute risk estimates. The ranking of absolute CVD risk is clearly essential for making collective decisions about treatment and preventive interventions in the population. This is rather important for the government/public health professionals, whose decision-making will be done based on such information alongside careful consideration on costs incurred and possible harms from screening and subsequent preventive interventions.

Additionally, risk assessment is also used as a tool to provide prognostic information or estimation of the possible absolute benefits from therapeutic/preventive interventions. In this case, accurate estimation of the absolute risk is crucial. An estimate of absolute CVD risk can inform the potential for absolute risk reduction, which provides patients with an idea of expected benefit from a therapy or intervention. This is more relevant to individual, rather than collective, decision-making. However, further research is needed to understand the process by which the clinician and patient interact once cardiovascular risk has been assessed. Providing CVD risk estimates to doctors and patients may improve perceived CVD risk and 


\begin{tabular}{ll}
\hline Rationale & Examples of utility of cardiovascular risk assessment in practice \\
\hline Risk stratification & $\begin{array}{l}\text { To rank individuals according to CVD risk and identify those at high risk for preventive } \\
\text { interventions }\end{array}$ \\
& $\begin{array}{l}\text { The Government makes decision if providing preventive intervention to a certain proportion } \\
\text { of the population is affordable and acceptable. }\end{array}$ \\
Prognostication & $\begin{array}{l}\text { To provide prognostic information to an individual, e.g. an individual is informed about how } \\
\text { likely he/she will develop cardiovascular disease in the next } 10 \text { years }\end{array}$ \\
& $\begin{array}{l}\text { To provide possible absolute benefits from therapeutic/preventive interventions, e.g. a doctor } \\
\text { can discuss with his/her patients about to what extent that their CVD risk will reduce if they } \\
\text { adhere to treatment and achieve certain therapeutic goals }\end{array}$ \\
Trimary prevention motivate an individual to modify health behaviours or adhere to treatment, i.e. when a \\
patient know smoking and high cholesterol contributes much to increased CVD risk, he/she \\
may be more encouraged to stop smoking, adopt healthy diet or regularly take statins \\
Monitoring CVD risk over time, e.g. information on favourable changes in absolute CVD risk \\
after an individual has adopted a healthy lifestyle will help enhance adherence to such a lifestyle.
\end{tabular}

Table 1. Overall rationale for cardiovascular risk assessment.

medical prescribing particularly in high-risk groups [18]; however, there is no strong evidence that a CVD risk assessment performed by a clinician helps to improve CVD-related health outcomes [8].

Finally, the principle justification for calculating cardiovascular risk as part of a preventive strategy is to motivate individuals to adhere to favourable behaviour modification and medical treatments. Apparently, two unchangeable risk factors, age and sex, contribute most to the predictive value of a CVD risk score. However, it may be difficult to persuade patients to change their behaviour using CVD risk scores that are mostly driven by risk factors that they cannot change. Risk scores which incorporate modifiable risk factors may be more useful for preventive strategies. That is, it may be important to use risk scores comprised of modifiable risk factors rather than using a score dominated by fixed parameters [7]. This approach is of particular interest as lifestyle risk factors, such as inadequate physical activity, poor dietary habits and adiposity, have been reported to deteriorate blood pressure, serum cholesterol and glucose-insulin homeostasis and also associated with novel cardiovascular risk factors such as endothelial function and inflammatory markers[19].

\section{Development and utility of CVD risk scores in developing countries}

Although cardiovascular risk assessment is recommended by many authorities, it has not been widely and routinely implemented in developing countries. This may be explained by a number of reasons. First, a small number of existing risk scores were originally developed in developing countries. Most CVD risk scores were derived from European descent populations in developed countries, whose background CVD risk may be significantly different from that in developing countries. For example, CVD risk scores developed in a relatively high-risk 
population, such as Framingham risk equations, will overestimate the absolute risk when applied to a novel population with a lower background CVD risk [20]. This phenomenon emphasizes that recalibration should be done before using such a risk score in a new population. However, a few developing countries have carried out recalibration of available risk scores partly because a small number of prospective cohorts are available in these countries and populations. Further, there is still debate over the cost-effectiveness of implementation of such an approach in developing countries. This may have affected policy decision concerning cardiovascular risk assessment in these resource-constrained countries.

A different approach was taken to overcome problems regarding the validity of using available risk scores in a novel population in developing countries. Using epidemiologic survey data from the Comparative Risk Assessment Project and the Asia Pacific Cohort Studies Collaboration, the WHO and International Society of Hypertension (ISH) developed CVD risk prediction charts specific to low- and medium-resource countries in 14 different WHO sub-regions, based on age, sex, systolic blood pressure, presence of type 2 diabetes mellitus, smoking status and total serum cholesterol levels (Figure 2) [21, 22]. They also developed a non-laboratory-based version of the risk prediction charts, which can be used in settings where measurement of blood cholesterol is not feasible.

Developing countries have different progresses in adopting the concept of cardiovascular risk assessment (Table 2). Some developing countries have only applied existing risk scores, namely Framingham risk equations, SCORE and WHO/ISH CVD risk prediction charts,

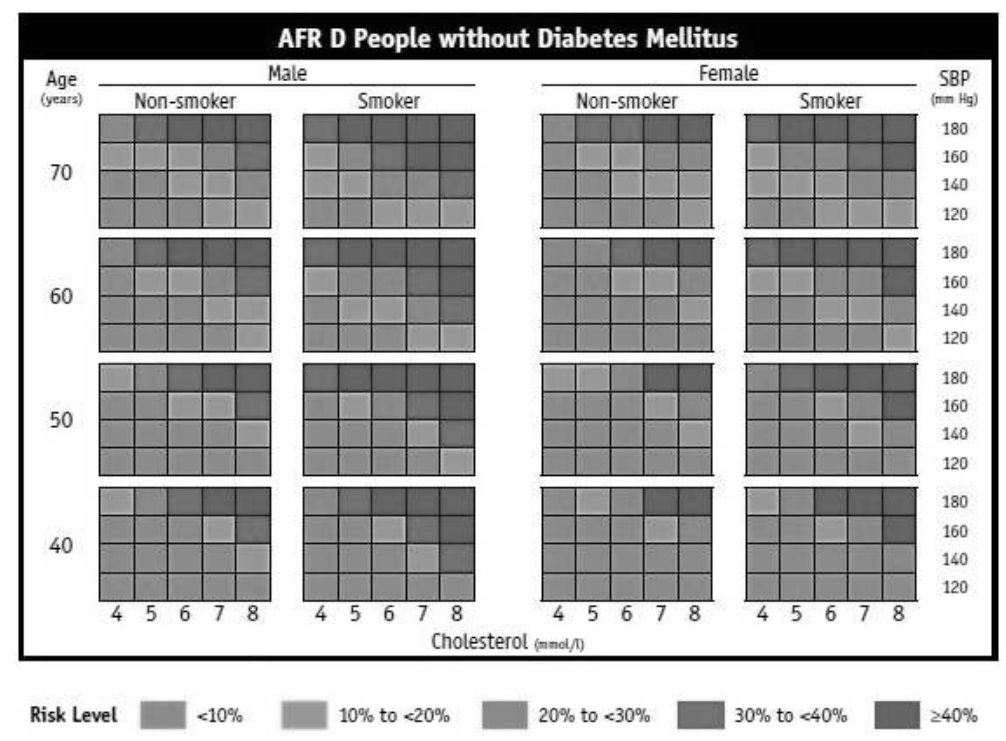

Figure 2. WHO/ISH risk prediction chart for Africa Sub-region D, 10-year risk of a fatal or non-fatal cardiovascular event by gender, age, systolic blood pressure, total blood cholesterol and smoking status in people without diabetes, Reproduced with approval from the WHO [22]. 


\section{Describing estimated CVD risk and Validation and recalibration of $\quad$ Developing a new CVD risk score the burden of high CVD risk in its existing risk scores in its population specific to its population population}

Saudi Arabia, Mozambique, Jamaica, Greece, Iran, Czech Republic, Poland, China, Thailand, Malaysia Pakistan, Sri Lanka Nepal, Mongolia, Lithuania, Russia

Cambodia

Table 2. Examples of developing countries that have adopted concept of cardiovascular risk assessment.

to cross-sectional risk factor data to obtain estimated CVD risk and describe the burden of high absolute CVD risk in the population. For example, low prevalences of high CVD risk according to the WHO/ISH CVD risk prediction charts were observed in many developing countries such as Saudi Arabia, Nigeria, Iran, Pakistan, Nepal, Sri Lanka and Cambodia, with lower than $10 \%$ of the populations being considered high CVD risk [21, 23].

Other countries carried out a validation study to see if the existing risk scores performed well in predicting cardiovascular events in its population and further recalibrated the risk scores [24-26]. For example, the office-based Framingham risk equations were recalibrated in 46,674 participants in the Golestan Cohort in North-East of Iran and the authors found that the risk score performed reasonably well in predicting CVD mortality (aROC of 0.76-0.79) and overestimated CVD risk in men [25]. The SCORE was validated against CVD mortality in Central and Eastern Europe and former Soviet Union countries, using prospective data from the WHO MONICA project and HAPIEE study. In the MONICA cohort, the SCORE showed only moderate discriminatory ability (aROC of 0.54-0.69) and good calibration in Czech Republic, Poland and Lithuania, but overestimation of fatal CVD in Russia [26].

A few developing countries have derived new CVD risk scores specific to their populations; these include China, Thailand and Malaysia. Using data on 9903 men and women participating in the USA-PRC Collaborative Study of Cardiovascular Epidemiology Cohort, a new CVD risk score was developed based on age, systolic blood pressure, serum total cholesterol, body mass index, current smoking status and diabetes mellitus [27]. The risk score was validated in another large Chinese cohort with good predictive ability. In Thailand, CHD risk score was developed using data from a cohort of workers of the Electric Generating Authority of Thailand, [28] and this multivariate risk score has been endorsed to use in patients with and without diabetes, and a simpler approach using presence or absence of CVD risk factors is recommended for population screening. Based on cost-effectiveness evidence, cardiovascular risk assessment using multivariate risk scores has recently been endorsed as part of the Universal Health Coverage Benefit Package [29]. However, there is no clear evidence about to what extent this approach has been adopted in clinical practice.

\section{Issues surrounding the utility of CVD risk scores}

Cardiovascular risk scores have been widely used in Western countries for almost 20 years and were introduced at a time when the cost of cholesterol-lowering drugs was an important 
political issue. International and national recommendations have specified cardiovascular risk thresholds that are considered justification for prescribing therapy. As these figures were based largely on the proportion of the population requiring treatment and the total cost of treatment, it is therefore uncertain if all health systems, particularly in developing countries, can afford such a policy.

\subsection{Laboratory- versus non-laboratory-based CVD risk assessment}

While most CVD risk assessment tools were based on a set of cardiovascular risk factors including laboratory factors such as cholesterol, a number of risk scores including simple or routinely available risk factors may be more relevant and applicable to developing countries, where laboratory testing is unavailable. Of note, it has been demonstrated in many studies that laboratory- and non-laboratory-based CVD risk assessments are similarly effective at predicting future cardiovascular disease [30-32]. A modelling study based on data from the EPIC-Norfolk cohort and published evidence on effectiveness of key preventive interventions clearly suggests that inviting individuals at high risk identified using routine data for a vascular risk assessment could prevent a similar number of new cardiovascular events with potential cost-saving, compared to inviting all individuals for laboratory-based CVD risk assessment [33]. Cost-effectiveness analyses confirm that single-stage or multi-stage nonlaboratory-based CVD risk assessment had a more attractive cost-effectiveness ratio or ICER than a laboratory-based Framingham risk-based approach, as reflected by lower estimated costs and higher QALYs [34].

\subsection{Are population-specific risk scores needed?}

The most commonly used cardiovascular risk score was originally developed in 5,573 men and women participating in the Framingham study in the early 1970s, which generally performs well in North America, but have reportedly performed less well in other populations. An alternative to attempting to find a universal risk score that will work in all populations may be to develop or recalibrate population-specific risk assessment tools.

Is it worth developing a new risk score which best suits with a new population or trying to improve the ability of the existing risk score to predict future disease events by including novel risk factors? The answer is likely to be 'No' for developing countries. Developing a new CVD risk score for developing countries or recalibrating existing risk scores in these countries may not be practical as it requires a prospective cohort to develop and validate the risk equations. A lack of prospective cohorts in developing countries will also limit the opportunities to recalibrate the existing risk scores in these countries/populations. Furthermore, including novel risk factors, such as biomarkers or genetic information, to a risk score with traditional CVD risk factors adds little to the predictive ability at disproportionately considerable costs $[35,36]$. This may be too expensive for clinical practice in both developed and developing countries. Furthermore, there is rarely evidence that reductions in any of these novel markers will lower cardiovascular risk [37]. Therefore, rather than attempting to develop a new risk assessment tool, it may be more beneficial to make sure that existing risk assessment tools are used more broadly and routinely throughout clinical practice. 
However, there remains a paradox of practice concerning CVD risk assessment in developing countries. While a population specific CVD risk score may be needed, a lack of prospective cohorts in developing countries prevent them from recalibrating available risk scores or developing a new one. While emphasis should be put on using existing risk scores more broadly and routinely, these risk scores seem to perform poorly in the developing countries and may lead to misclassification of individuals who do and do not require treatments.

\subsection{Is universal CVD risk assessment in developing countries cost-effective?}

As screening for high-risk individuals using CVD risk scores always comes with costs related to screening itself and subsequent preventive interventions, whether it is cost-effective in settings of resource-scarce countries is an important concern. With limited resources, it might be sensible to focus preventive efforts on those who will benefit most. Furthermore, the ratio of costs to benefits will be more favourable in individuals where the benefits are larger and this approach usually leads to manageable numbers of individuals.

CVD risk stratification will enable the government to use resources in a highly efficient way. Different thresholds for starting intensive preventive interventions may be applied to countries with different resources. For example, the WHO recommends that a 10 -year CVD risk threshold of 30-40\% may be used in medium- and low-resource countries [38]. When applying a WHO-ISH CVD risk prediction charts to randomly selected individuals from a number of developing countries such as Nigeria, Iran, China, Pakistan, Georgia, Nepal, Cuba and Sri Lanka, adopting WHO recommended 30\% CVD risk threshold would help reduce healthcare expenditure by avoiding unnecessary drug treatment and thus reducing drug costs [21]. As equipment and facilities for CVD risk screening in developing countries may be limited, the WHO and International Society of Hypertension in 2002 proposed a simple approach to cardiovascular risk stratification based on history, blood pressure measurement and selective urine analysis for those with systolic/diastolic blood pressure of $\geq 140 / 90 \mathrm{mmHg}$ [39]. Also, the WHO proposed different scenarios of CVD risk management which may be suitable to set with different diagnostic and therapeutic facilities and resources (Table 3 ).

However, it remains uncertain if universal CVD risk screening is cost-effective for resourceconstrained settings in developing countries. In Thailand, cardiovascular risk assessment using a population-specific risk score is recommended for men and women aged 35 years and above at five-year intervals. The corresponding budget impact analysis shows that such an approach was financially feasible for the Thai setting, with the incremental cost-effectiveness ratio below the country's ceiling threshold of USD 4530/QALY [29]. In the contrary, a modelling study in Malaysian population suggests that universal screening would result in screening an additional 7169 individuals, with an incremental cost of USD 115,033 for detection of one additional high-risk individual in comparison to targeted screening of those aged 35 years and above [40]. Further, incremental cost and impact of detection of high-risk individuals would be higher for women than men for both universal screening and screening specific age groups. The authors suggested that targeted gender- and age-specific screening strategies would ensure more optimal use of limited resources compared to the country's policy recommendations of universal screening [40]. 


\begin{tabular}{|c|c|c|c|}
\hline Resource availability & Scenario 1 & Scenario 2 & Scenario 3 \\
\hline Human resource & $\begin{array}{l}\text { Non-physician health } \\
\text { workers }\end{array}$ & $\begin{array}{l}\text { Medical doctor or specially } \\
\text { trained nurse }\end{array}$ & $\begin{array}{l}\text { Medical doctor with access to full } \\
\text { specialist care }\end{array}$ \\
\hline Equipment & $\begin{array}{l}\text { Stethoscope, blood } \\
\text { pressure measurement } \\
\text { device, measuring tape or } \\
\text { weighing scale } \\
\text { Optional: test tubes, holder, } \\
\text { burner, solution or test strips } \\
\text { for checking urine glucose }\end{array}$ & $\begin{array}{l}\text { Stethoscope, blood } \\
\text { pressure measurement } \\
\text { device, tape and weighing } \\
\text { scale, test tubes, holder, } \\
\text { burner, solution or test } \\
\text { strips for checking urine } \\
\text { glucose and albumin }\end{array}$ & $\begin{array}{l}\text { Stethoscope, blood } \\
\text { pressure measurement } \\
\text { device, tape and weighing } \\
\text { scale, electrocardiograph, } \\
\text { ophthalmoscope, urine analysis, } \\
\text { blood analysis: fasting blood } \\
\text { sugar, electrolytes, creatinine, } \\
\text { cholesterol and lipoproteins }\end{array}$ \\
\hline Generic drugs & $\begin{array}{l}\text { Essential: thiazide diuretics } \\
\text { Optional: metformin (for } \\
\text { refill) }\end{array}$ & $\begin{array}{l}\text { Thiazide diuretics, Beta } \\
\text { blockers, Angiotensin } \\
\text { converting enzyme } \\
\text { inhibitors, calcium channel } \\
\text { blockers (sustained release } \\
\text { formulations) (Reserpine } \\
\text { and methyldopa if the } \\
\text { above anti-hypertensives } \\
\text { are unavailable), Aspirin, } \\
\text { Metformin (for refill) }\end{array}$ & $\begin{array}{l}\text { Thiazide diuretics, Beta blockers, } \\
\text { Angiotensin converting enzyme } \\
\text { inhibitors, calcium channel } \\
\text { blockers (sustained release } \\
\text { formulations) (Reserpine and } \\
\text { methyldopa if the above anti- } \\
\text { hypertensives are unavailable), } \\
\text { Aspirin, Insulin, Metformin, } \\
\text { Glibenclamide, Statins (if } \\
\text { affordable) Angiotensin receptor } \\
\text { blocker (if affordable) }\end{array}$ \\
\hline
\end{tabular}

Table 3. Three scenarios for CVD risk assessment for setting with different resource availability, adapted from WHO CVD risk management package for low and medium resource settings [38].

\subsection{Preventive interventions following CVD risk assessment}

There are challenges concerning preventive interventions following CVD risk assessment. Different preventive interventions, ranging from lifestyle modification to medication, are recommended for individuals or groups with different levels of absolute CVD risk. For example, healthy diet, adequate physical activity and weight control should be encouraged in all individuals, and monitoring of CVD risk profiles every 3-6 months, nicotine replacement therapy, giving statins in those with an absolute 10 -year risk of $\geq 20 \%$, and prescribing antiplatelet drugs in those with a 10 -year risk of $\geq 30 \%$ [41]. However, there remains unclear whether such differential interventions can be implemented in developing countries, where basic infrastructure and resources for preventive interventions in primary care may be lacking.

\section{Limitations and implications for alternative approaches}

Although identifying individuals or groups at high risk using multivariate CVD risk scores is beneficial, the 'population-based' strategy, which aims at reducing risk in the entire population, regardless of each individual's level of risk and potential benefits, may also be needed. Ability of CVD risk scores to identify individuals at high risk is a key to effective high-risk strategies. It may be more effective to concentrate our efforts and resources where the need, and therefore the benefit, is likely to be greatest. Interventions are also 
matched well with the needs of individuals, probably resulting in better motivation and compliance to medications or behaviour change [42]. However, the benefits of the highrisk approach are limited to a minority of the population. Further, a high-risk strategy demands that individuals change eating, smoking and physical activity habits that may be largely shaped and constrained by social norms (e.g. to eat differently from family and friends), and may thus be seen as 'behaviourally inappropriate'. As this approach does not seek to address the underlying causes of health problems in the population, it is only palliative and temporary [43]. The high-risk approach was described by Geoffrey Rose as 'no more than an expensive rescue operation, offering disappointingly little towards solving the overall problem', so it cannot be the sole means for prevention of cardiovascular disease [42].

Given compelling evidence that more cases of cardiovascular disease arise from a larger number of people at low risk than the smaller number of people at high risk, it might be more beneficial to shift the whole distribution of risk factors, such as body mass index, cholesterol or blood glucose, in the population in a favourable direction (population-based strategy). This phenomenon is commonly observed for diseases where the association of the disease with risk factors is linear or curvilinear, while the population distribution of these risk factors is approximately normal. For example, Emberson et al. demonstrated that small reductions in the population distribution of cardiovascular risk factors (total cholesterol and systolic blood pressure) might prevent similar or more CVD events than a strategy focusing preventive efforts on those at highest risk [44]. Therefore, in addition to a high-risk approach, there may be an important role for a 'population-based approach' to shift the population distribution of CVD risk factors.

In practice, balanced implementation of the high-risk and population-based approaches for CVD prevention is likely to be necessary [2]. Within resource-constrained health service systems in developing countries, the high-risk preventive strategies, which focus efforts on those at highest risk, may be seen as a feasible and cost-effective means of prevention. However, complementary population-based preventive strategies are also needed to address the cause of the disease incidence in the population.

In conclusion, CVD risk stratification in developing countries will assure that limited resource be allocated to individuals or groups who need it most. In developing countries, the clear rationale for cardiovascular risk assessment is crucial. The main rationale for cardiovascular risk assessment includes ranking individuals according to absolute cardiovascular risk for the purpose of targeting therapy to those at greatest risk, providing prognostic information or accurate estimation of the likely benefits from preventive/therapeutic interventions, and motivating individuals to change their behaviours and adhere to treatments. As not many developing countries have adopted this approach, a number of issues need to be addressed, including development of population-specific risk scores, recalibration of available risk scores and uncertainty over cost-effectiveness of CVD risk assessment in developing countries. Although this high-risk approach appears to be effective and practical for developing countries, a complementary population-based approach is needed to maximize benefits for CVD prevention. 


\section{Author details}

Parinya Chamnan ${ }^{1 *}$ and Wichai Aekplakorn ${ }^{2}$

*Address all correspondence to: parinya.chamnan@cardiomet-res.org

1 Cardiometabolic Research Group, Department of Social Medicine, Sanpasitthiprasong Hospital,Ubon Ratchathani, Thailand

2 Department of Community Medicine, Faculty of Medicine, Ramathibodi Hospital, Mahidol University, Bangkok, Thailand

\section{References}

[1] Lloyd-Jones DM. Cardiovascular risk prediction: Basic concepts, current status, and future directions. Circulation. 2010;121(15):1768-1777

[2] Hingorani AD, Psaty BM. Primary prevention of cardiovascular disease: Time to get more or less personal? Journal of the American Medical Association. 2009;302(19):2144-2145

[3] Piepoli MF, Hoes AW, Agewall S, Albus C, Brotons C, Catapano AL, et al. 2016 European Guidelines on cardiovascular disease prevention in clinical practice: The sixth joint task force of the european society of cardiology and other societies on cardiovascular disease prevention in clinical practice (constituted by representatives of 10 societies and by invited experts) developed with the special contribution of the european association for cardiovascular prevention \& rehabilitation (EACPR). European Heart Journal. 2016;37(29):2315-2381

[4] Prepared by JBS 2. Joint British Societies' guidelines on prevention of cardiovascular disease in clinical practice. Heart. 2005;91(Suppl 5):v1-v52

[5] Prepared by Third report of the national cholesterol education program (NCEP). Expert panel on detection, evaluation, and treatment of high blood cholesterol in adults (Adult Treatment Panel III) final report. Circulation. 2002;106(25):3143-3421

[6] Goff Jr, DC, Lloyd-Jones DM, Bennett G, Coady S, D'Agostino RB, Gibbons R, et al. 2013 ACC/AHA guideline on the assessment of cardiovascular risk: A report of the American College of Cardiology/American Heart Association Task Force on practice guidelines. Circulation. 2014;129(25 Suppl 2):S49-S73

[7] Chamnan P, Simmons RK, Sharp SJ, Griffin SJ, Wareham NJ. Cardiovascular risk assessment scores for people with diabetes: A systematic review. Diabetologia. 2009;52(10): 2001-2014

[8] Brindle P, Beswick A, Fahey T, Ebrahim S. Accuracy and impact of risk assessment in the primary prevention of cardiovascular disease: A systematic review. Heart. 2006;92(12): 1752-1759 
[9] Prospective Studies Collaboration, Lewington S, Whitlock G, Clarke R, Sherliker P, Emberson J. et al. Blood cholesterol and vascular mortality by age, sex, and blood pressure: A meta-analysis of individual data from 61 prospective studies with 55,000 vascular deaths. Lancet. 2007;370(9602):1829-1839

[10] Chamnan P, Simmons RK, Jackson R, Khaw KT, Wareham NJ, Griffin SJ. Non-diabetic hyperglycaemia and cardiovascular risk: Moving beyond categorisation to individual interpretation of absolute risk. Diabetologia. 2011;54(2):291-299

[11] Lewington S, Clarke R, Qizilbash N, Peto R, Collins R, Prospective Studies C. Age-specific relevance of usual blood pressure to vascular mortality: A meta-analysis of individual data for one million adults in 61 prospective studies. Lancet. 2002;360(9349):1903-1913

[12] Jackson R, Lawes CM, Bennett DA, Milne RJ, Rodgers A. Treatment with drugs to lower blood pressure and blood cholesterol based on an individual's absolute cardiovascular risk. Lancet. 2005;365(9457):434-441

[13] Neaton JD, Wentworth D. Serum cholesterol, blood pressure, cigarette smoking, and death from coronary heart disease. Overall findings and differences by age for 316,099 white men. Multiple risk factor intervention trial research group. Archives of Internal Medicine. 1992;152(1):56-64

[14] Baigent C, Keech A, Kearney PM, Blackwell L, Buck G, Pollicino C, et al. Efficacy and safety of cholesterol-lowering treatment: Prospective meta-analysis of data from 90,056 participants in 14 randomised trials of statins. Lancet. 2005;366(9493):1267-1278

[15] Yusuf S, Sleight P, Pogue J, Bosch J, Davies R, Dagenais G. Effects of an angiotensin-converting-enzyme inhibitor, ramipril, on cardiovascular events in high-risk patients. The heart outcomes prevention evaluation study investigators. The New England Journal of Medicine. 2000;342(3):145-153

[16] Jackson R. Cardiovascular risk prediction: are we there yet? Heart. 2008;94(1):1-3

[17] Ndindjock R, Gedeon J, Mendis S, Paccaud F, Bovet P. Potential impact of single-riskfactor versus total risk management for the prevention of cardiovascular events in Seychelles. Bulletin of the World Health Organization. 2011;89(4):286-295

[18] Usher-Smith JA, Silarova B, Schuit E, Moons KG, Griffin SJ. Impact of provision of cardiovascular disease risk estimates to healthcare professionals and patients: A systematic review. British Medical Journal Open. 2015;5(10):e008717

[19] Mozaffarian D, Wilson PW, Kannel WB. Beyond established and novel risk factors: lifestyle risk factors for cardiovascular disease. Circulation. 2008;117(23):3031-3038

[20] Liu J, Hong Y, D'Agostino RB, Sr, Wu Z, Wang W, Sun J, et al. Predictive value for the Chinese population of the Framingham CHD risk assessment tool compared with the Chinese multi-provincial cohort study. Journal of the American Medical Association. 2004;291(21):2591-2599 
[21] Mendis S, Lindholm LH, Anderson SG, Alwan A, Koju R, Onwubere BJ, et al. Total cardiovascular risk approach to improve efficiency of cardiovascular prevention in resource constrain settings. Journal of Clinical Epidemiology. 2011;64(12):1451-1462

[22] Mendis S, Lindholm LH, Mancia G, Whitworth J, Alderman M, Lim S, et al. World Health Organization (WHO) and International Society of Hypertension (ISH) risk prediction charts: Assessment of cardiovascular risk for prevention and control of cardiovascular disease in low and middle-income countries. Journal of Hypertension. 2007;25(8):1578-1582

[23] Otgontuya D, Oum S, Buckley BS, Bonita R. Assessment of total cardiovascular risk using WHO/ISH risk prediction charts in three low and middle income countries in Asia. BMC Public Health. 2013;13:539

[24] Panagiotakos DB, Georgousopoulou EN, Fitzgerald AP, Pitsavos C, Stefanadis C. Validation of the HellenicSCORE (a Calibration of the ESC SCORE Project) regarding 10-Year risk of fatal cardiovascular disease in Greece. Hellenic Journal of Cardiology. 2015;56(4):302-308

[25] Sepanlou SG, Malekzadeh R, Poustchi H, Sharafkhah M, Ghodsi S, Malekzadeh F, et al. The clinical performance of an office-based risk scoring system for fatal cardiovascular diseases in North-East of Iran. PLoS One. 2015;10(5):e0126779

[26] Vikhireva O, Pajak A, Broda G, Malyutina S, Tamosiunas A, Kubinova R, et al. SCORE performance in Central and Eastern Europe and former Soviet Union: MONICA and HAPIEE results. European Heart Journal. 2014;35(9):571-577

[27] Wu Y, Liu X, Li X, Li Y, Zhao L, Chen Z, et al. Estimation of 10-year risk of fatal and nonfatal ischemic cardiovascular diseases in Chinese adults. Circulation. 2006;114(21):2217-2225

[28] Yamwong S. Development of Thai CVD risk score. Public Health Foundation and Thailand Research Funds, Bangkok. 2005

[29] Teerawattananon Y, Kingkaew P, Koopitakkajorn T, Youngkong S, Tritasavit N, Srisuwan $\mathrm{P}$, et al. Development of a health screening package under the universal health coverage: The role of health technology assessment. Health Economics. 2016;25(Suppl 1):162-178

[30] Gaziano TA, Young CR, Fitzmaurice G, Atwood S, Gaziano JM. Laboratory-based versus non-laboratory-based method for assessment of cardiovascular disease risk: The NHANES I follow-up study cohort. Lancet. 2008;371(9616):923-931

[31] D'Agostino RB, Sr., Vasan RS, Pencina MJ, Wolf PA, Cobain M, Massaro JM, et al. General cardiovascular risk profile for use in primary care: The Framingham heart study. Circulation. 2008;117(6):743-753

[32] Chamnan P, Simmons RK, Hori H, Sharp S, Khaw KT, Wareham NJ, et al. A simple risk score using routine data for predicting cardiovascular disease in primary care. British Journal of General Practice. 2010;60(577):e327-e334 
[33] Chamnan P, Simmons RK, Khaw KT, Wareham NJ, Griffin SJ. Estimating the population impact of screening strategies for identifying and treating people at high risk of cardiovascular disease: Modelling study. British Medical Journal. 2010;340:c1693

[34] Pandya A, Weinstein MC, Salomon JA, Cutler D, Gaziano TA. Who needs laboratories and who needs statins?: Comparative and cost-effectiveness analyses of non-laboratorybased, laboratory-based, and staged primary cardiovascular disease screening guidelines. Circulation: Cardiovascular Quality and Outcomes. 2014;7(1):25-32

[35] Lippi G, Salvagno GL, Targher G, Guidi GC. Multiple biomarkers for the prediction of first major cardiovascular events and death: Considerable costs and limited benefits. Medscape General Medicine. 2007;9(1):34

[36] Wang TJ, Gona P, Larson MG, Tofler GH, Levy D, Newton-Cheh C, et al. Multiple biomarkers for the prediction of first major cardiovascular events and death. The New England Journal of Medicine. 2006;355(25):2631-2639

[37] Ridker PM, Brown NJ, Vaughan DE, Harrison DG, Mehta JL. Established and emerging plasma biomarkers in the prediction of first atherothrombotic events. Circulation. 2004;109(25 Suppl 1):IV6-IV19

[38] Wolrd Health Organization. WHO CVD-risk management package for low- and medium resource settings. Switzerland: Wolrd Health Organization; 2002

[39] Chalmers J, World Health Organization International - Society of H. Enhancing risk stratification in hypertensive subjects: How far should we go in routine screening for target organ damage? Journal of Hypertension. 2002;20(7):1255-1257

[40] Selvarajah S, Haniff J, Kaur G, Guat Hiong T, Bujang A, Chee Cheong K, et al. Identification of effective screening strategies for cardiovascular disease prevention in a developing country: Using cardiovascular risk-estimation and risk-reduction tools for policy recommendations. BMC Cardiovascular Disorders. 2013;13:10

[41] World Health Organization. Prevention of cardiovascular disease: Guidelines for assessment and managemnet of cardiovascular risk. Geneva: World Health Organization; 2007

[42] Rose G. Rose's strategy of preventive medicine. New York: Oxford University Press; 2008

[43] Rose G. Sick individuals and sick populations. International Journal of Epidemiology. 2001;30(3):427-432; discussion 33-34

[44] Emberson J, Whincup P, Morris R, Walker M, Ebrahim S. Evaluating the impact of population and high-risk strategies for the primary prevention of cardiovascular disease. European Heart Journal. 2004;25(6):484-491 